\title{
Dynamical Behavior for a Food-Chain Model with Impulsive Harvest and Digest Delay
}

\author{
Baodan Tian, ${ }^{1,2}$ Shouming Zhong, ${ }^{1}$ Ning Chen, ${ }^{2}$ and Yanhong Qiu ${ }^{2}$ \\ ${ }^{1}$ School of Mathematical Sciences, University of Electronic Science and Technology of China, Chengdu 611731, China \\ ${ }^{2}$ School of Science, Southwest University of Science and Technology, Mianyang 621010, China \\ Correspondence should be addressed to Baodan Tian; tianbaodan@swust.edu.cn
}

Received 27 April 2014; Revised 6 August 2014; Accepted 7 August 2014; Published 25 August 2014

Academic Editor: Alexander Timokha

Copyright (c) 2014 Baodan Tian et al. This is an open access article distributed under the Creative Commons Attribution License, which permits unrestricted use, distribution, and reproduction in any medium, provided the original work is properly cited.

\begin{abstract}
We investigate the dynamics of a food-chain system with digest delay and periodic harvesting for the prey. By using the comparison theorem, small amplitude skills in the impulsive differential equation, and a special qualitative analysis method in the delay differential equation, we prove that there exists a predators-eradication periodic solution which is globally attractive and show that the pest population can be controlled under the economic threshold level and the system can be uniformly permanent when the harvest period $T$ is long enough or the harvesting rate $\delta$ is not too large. Furthermore, we perform a series of numerical simulations to display the effects of the digest delay and periodic harvesting on the dynamic behavior of the food-chain system.
\end{abstract}

\section{Introduction}

It is now widely believed that pest outbreaks often cause serious ecological and economic problems. As a result, ecologists and mathematics acknowledge the importance of controlling insect pests of agriculture and insect vectors of plant [1]. Integrated pest management involves choosing appropriate tactics from a range of pest control techniques including biological, cultural, and chemical methods to suit individual cropping systems, pest complexes, and local environments [2-4]. For example, as concerning the chemical control strategy, it seems to be quick and efficient to decrease the pests population by the chemical insecticides in a short time. But when we use excess of chemical insecticides to kill the pest population, not only is the environment polluted, but also the natural enemies (or beneficial species) will be killed at the same time, even leading to the adaptability of the pests and the ineffectiveness of the insecticides. And this will lead to the waste of the manpower and material resources and we cannot reach our expected results, even bringing negative effects. And as concerning the biological control strategy, that is, stocking the natural enemies periodically by artificial culture or immigration, we can avoid many human losses caused by environmental pollution in this way, while it will take us a long time and a complex process for the culture of the natural enemies. Therefore, it is important to establish mathematical models to provide valuable information about how to control pest outbreaks, especially to study the dynamical behavior of the pests and their natural enemies.

On the other hand, when the prey-predator system is referred, sometimes there is a digest and absorption time (which is the so-called digest delay) during the predation instead of translating the food into growth rate immediately. Hence, in order to model the relationship between the predator and the prey more accurately, it is more reasonable to introduce time delay into the model. Usually, there are two kinds of delays in the ecological model, that is, discrete timedelay and distributed time-delay (continuous time delay). Recently, it seems that much more attention is paid on the models with impulsive perturbations and time delay [5-13], and some of them [5-8] trend to focus on the impulsive model with distributed time-delay, in which a kernel function 
$F(t)=a e^{-a t}, a>0$. To the best of our knowledge, the study on the effect of the discrete time-delay on the impulsive system seems to be rare.

Recently, in an effort to seek more efficient pest management strategies, Yu et al. [14] considered an ecological model with impulsive control strategy as follows:

$$
\begin{aligned}
& \frac{d x}{d t}=r_{1} x(t)\left(1-\frac{x(t)}{k_{1}}\right)-\frac{a_{1} x(t) y(t)}{b_{1}+x(t)}-\frac{a_{2} x(t) z(t)}{b_{2}+x(t)}, \\
& \frac{d y}{d t}=r_{2} y(t)\left(1-\frac{y(t)}{k_{2}}\right)+\frac{e_{1} a_{1} x(t) y(t)}{b_{1}+x(t)}-\frac{a_{3} y(t) z(t)}{b_{3}+y(t)}, \\
& \frac{d z}{d t}=\frac{e_{2} a_{2} x(t) z(t)}{b_{2}+x(t)}+\frac{e_{3} a_{3} y(t) z(t)}{b_{3}+y(t)}-m z(t), \\
& \Delta x(t)=0, \quad t \neq n T, \quad n \in N^{*}, \\
& \Delta y(t)=0, \quad \Delta z(t)=p, \\
& t=n T, \quad n \in N^{*},
\end{aligned}
$$

where $x(t), y(t)$, and $z(t)$ are the densities of one prey and two predators at time $t$, respectively, and $\Delta x(t)=x\left(t^{+}\right)-x(t)$, $\Delta y(t)=y\left(t^{+}\right)-y(t)$, and $\Delta z(t)=z\left(t^{+}\right)-z(t) . r_{i}(i=1,2)$ are the intrinsic growth rate, $a_{i}(i=1,2,3)$ and $b_{i}(i=$ $1,2)$ measure the efficiency of the prey in evading a predator attack, and $b_{3}$ has similar meaning as that of $b_{i} \cdot e_{i}(i=1,2,3)$ denote the efficiency with which resources are converted to new consumers, $k_{i}(i=1,2)$ are carrying capacity in the absence of predator, $m$ is the mortality rates for the predator, $T$ is the period of the impulsive effect, $n \in N, N$ is the set of all nonnegative integers, and $p>0$ is the release amount of predator at $t=n T$.

In [14], the authors studied the food-chain prey-predator model (1) with periodic release on the higher predator (enemy population) $z(t)$ and discussed some efficient biological control strategies for the system. But they had not considered the affection of the digest delay.

Based on the discussions above, we consider the following food-chain prey-predator model with periodic harvest on the prey (the pest population) $x(t)$, but the lower predator $y(t)$ only lives on the prey. That is, if the prey $x(t)$ is extinct, the lower predator $y(t)$ has no other food resources, and it is inevitable to be extinct. Furthermore, we assume that there is a digest and absorption time $\tau$ during the predation of the higher predator $z(t)$ instead of translating the food into growth rate immediately, and the final model we will study in this paper is as follows:

$$
\begin{aligned}
& \frac{d x}{d t}=x(t)\left(r_{1}-d_{1} x(t)\right)-\frac{a_{1} x(t) y(t)}{b_{1}+x(t)}-\frac{a_{2} x(t) z(t)}{b_{2}+x(t)}, \\
& \frac{d y}{d t}=\frac{e_{1} a_{1} x(t) y(t)}{b_{1}+x(t)}-\frac{a_{3} y(t) z(t)}{b_{3}+y(t)}-r_{2} y(t)-d_{2} y^{2}(t), \\
& \frac{d z}{d t}=\frac{e_{2} a_{2} x(t-\tau) z(t-\tau)}{b_{2}+x(t-\tau)}
\end{aligned}
$$

$$
\begin{gathered}
+\frac{e_{3} a_{3} y(t-\tau) z(t-\tau)}{b_{3}+y(t-\tau)}-m z(t), \\
t \neq n T, \quad n \in N^{*}, \\
\Delta x(t)=-\delta x(t), \quad \Delta y(t)=0, \quad \Delta z(t)=0, \\
t=n T, \quad n \in N^{*},
\end{gathered}
$$

where $d_{1}, d_{2}>0$ are the coefficients of density dependence of $x(t)$ and $y(t)$; since the higher predator population always have stronger ability to migrate, then it is more possible for them to escape from the inner competition. Thus, the impact of density dependence is relatively small, so we do not consider the density dependence of higher predator $z(t)$ in the model. Further, $r_{1}$ is the intrinsic increasing rate of the prey population $x(t), r_{2}$ is the death rate of the lower predator $y(t), a_{i}$ and $b_{i}(i=1,2,3)$ measure the efficiency of the prey in evading a predator attack. $e_{i}(i=1,2,3)$ denote the efficiency that resources are converted to the new consumers, and $m$ is the mortality rates of the higher predator. $0<\delta<1$ is the harvesting rate at the periodic time $t=n T\left(n \in N^{*}\right)$, and the initial condition for system (2) is

$$
\begin{array}{r}
\left(\varphi_{1}(s), \varphi_{2}(s), \varphi_{3}(s)\right) \in C_{+}=C\left([-\tau, 0], R_{+}^{3}\right), \\
\varphi_{i}(0)>0, \quad i=1,2,3 .
\end{array}
$$

From the viewpoint of ecological meanings, we only consider system (2) in the nonnegative region $D=\{(x, y, z) \mid$ $x \geq 0, y \geq 0, z \geq 0\}$.

The rest of this paper is organized as follows: in Section 2, we will give some basic definitions and several useful lemmas for the proof of our main results. In Section 3, we will state and prove our main results such as boundedness of the solution, global attractivity of the predators-eradication periodic solution, and sufficient conditions for the permanence of the system. In Section 4, we give some numerical examples to support our theoretical results. And in the last section, we provide a brief discussion and the summary of our main results.

\section{Preliminaries}

Let $R_{+}=[0,+\infty), R_{+}^{3}=\left\{(x, y, z) \in R_{+}^{3} \mid x \geq 0, y \geq\right.$ $0, z \geq 0\}, \Omega=\operatorname{int} R_{+}^{3}, N$ be the set of all nonnegative integers. Denote as $f=\left(f_{1}, f_{2}, f_{3}\right)$ the map defined by the right-hand side of the first, second, and third equation of the system (2).

Let $V: R_{+} \times R_{+}^{3} \rightarrow R_{+}$; then $V$ is said to belong class $V_{0}$ if

(1) $V$ is continuous in $(n T,(n+1) T] \times R_{+}^{3}$, and for each $x \in R_{+}^{3}, n \in N^{*}$,

$$
\lim _{(t, y) \rightarrow\left(n T^{+}, x\right)} V(t, y)=V\left(n T^{+}, x\right) \text { exists; }
$$

(2) $V$ is locally Lipschitzian for $x$. 
Definition 1. $V \in V_{0}$; then for $(t, x) \in(n T,(n+1) T] \times R_{+}^{3}$, the upper right derivative of $V(t, x)$ with respect to the system (2) is defined as

$$
D^{+} V(t, x)=\lim _{h \rightarrow 0^{+}} \sup \frac{V(t+h, x+h f(t, x))-V(t, x)}{h} .
$$

Definition 2. System (2) is said to be permanent if there exist positive $m_{i}, M_{i}(i=1,2,3)$ and $T_{0}$, such that for any $t>T_{0}$, each positive solution $X(t)=(x(t), y(t), z(t))$ of system (2) satisfies with

$$
\begin{gathered}
m_{1} \leq x(t) \leq M_{1}, \quad m_{2} \leq y(t) \leq M_{2}, \\
m_{3} \leq z(t) \leq M_{3} .
\end{gathered}
$$

The solution $X(t)$ of system (2) is a piecewise continuous function. $X: R_{+} \rightarrow R_{+}^{3}$ is continuous on $(n T,(n+1) T]$, and $X\left(n T^{+}\right)=\lim _{t \rightarrow n T^{+}} X(t)$ exists, where $n \in N$. The smoothness properties of $f$ guarantee the global existence and the uniqueness of solution of system (2); more details can be seen in the books $[15,16]$.

Also, we will use the following comparison theorem of impulsive differential equation (see, [15]).

Lemma 3. Suppose $V \in V_{0}, X\left(t_{0}\right)=X_{0}$, and assume that

$$
\begin{gathered}
D^{+} V(t, X(t)) \leq(\geq) g(t, V(t, X(t))), \quad t \neq n T, \\
V\left(t, X\left(t^{+}\right)\right) \leq(\geq) \psi_{n}(V(t, X(t))), \quad t=n T,
\end{gathered}
$$

where $g: R_{+} \times R_{+} \rightarrow R$ is continuous in $(n T,(n+1) T] \times R_{+}$and for each $u \in R_{+}, n \in N, \lim _{(t, y) \rightarrow\left(n T^{+}, u\right)} g(t, y)=g\left(n T^{+}, u\right)$ exists, $\psi_{n}: R_{+} \rightarrow R_{+}$is nondecreasing.

Let $r(t)$ be the maximal (minimal) solution of the scalar impulsive differential equation

$$
\begin{gathered}
\frac{d u}{d t}=g(t, u(t)), \quad t \neq n T, \\
u\left(t^{+}\right)=\psi_{n}(u(t)), \quad t=n T, \\
u\left(0^{+}\right)=u_{0} .
\end{gathered}
$$

existing on $[0,+\infty)$. Then $V\left(0^{+}, X_{0}\right) \leq(\geq) u_{0}$ implies that $V(t, X(t)) \leq(\geq) r(t), t \geq 0$, where $X(t)$ is any solution of $(2)$.

Lemma 4 (see [17]). Consider the following delay differential equation:

$$
\frac{d x}{d t}=r_{1} x(t-\tau)-r_{2} x(t)
$$

where $r_{1}, r_{2}$, and $\tau$ are all positive constants and $x(t)>0$ for all $t \in[-\tau, 0]$.

(1) If $r_{1}<r_{2}$, then $\lim _{t \rightarrow \infty} x(t)=0$.

(2) If $r_{1}>r_{2}$, then $\lim _{t \rightarrow \infty} x(t)=+\infty$.

In order to discuss the predators-eradication periodic solution of system (2) in the next section, we will give some basic properties about the following subsystem of system (2) at first:

$$
\begin{gathered}
\frac{d x}{d t}=x(t)\left(r_{1}-d_{1} x(t)\right), \quad t \neq n T, \\
x\left(t^{+}\right)=(1-\delta) x(t), \quad t=n T, n \in N^{*}, \\
x\left(0^{+}\right)=x_{0} .
\end{gathered}
$$

It is easy to solve above system (10) between pulses, yielding

$$
\begin{array}{r}
x(t)=\frac{r_{1} x\left(n T^{+}\right)}{d_{1} x\left(n T^{+}\right)+\left[r_{1}-d_{1} x\left(n T^{+}\right)\right] \exp \left(-r_{1}(t-n T)\right)}, \\
t \in(n T,(n+1) T], \quad n \in N^{*} .
\end{array}
$$

Then we can obtain the stroboscopic map of (11) as follows:

$$
\begin{aligned}
x\left((n+1) T^{+}\right) & =\frac{r_{1}(1-\delta) x\left(n T^{+}\right)}{d_{1} x\left(n T^{+}\right)+\left[r_{1}-d_{1} x\left(n T^{+}\right)\right] \exp \left(-r_{1} T\right)} \\
& \triangleq F\left(x\left(n T^{+}\right)\right) .
\end{aligned}
$$

If we denote $u=x\left(n T^{+}\right)$, then

$$
F(u)=\frac{r_{1}(1-\delta) u}{d_{1} u+\left(r_{1}-d_{1} u\right) \exp \left(-r_{1} T\right)},
$$

which has two fixed points:

$$
u_{1}^{*}=0, \quad u_{2}^{*}=\frac{r_{1}\left(1-\delta-\exp \left(-r_{1} T\right)\right)}{d_{1}\left(1-\exp \left(-r_{1} T\right)\right)} \text {. }
$$

Then we have Lemma 5 for the subsystem (10) by the method in [9].

Lemma 5. Suppose $\delta^{*}=1-e^{-r_{1} T}$, and then we have the following results.

(1) If $\delta>\delta^{*}$, then the trivial periodic solution of system (10) is globally asymptotically stable.

(2) If $\delta<\delta^{*}$, then system (10) has a unique positive periodic solution $x^{*}(t)$ which is globally asymptotically stable.

Proof. If $\delta>\delta^{*}$, we can see that the stroboscopic map of (11) has a unique trivial fixed point $u_{1}^{*}=0$, and by a direct calculation we can obtain

$$
\left|\frac{d F}{d u}\right|_{u=u_{1}^{*}}=\frac{1-\delta}{e^{-r_{1} T}}<1 .
$$

Hence, the trivial periodic solution $u_{1}^{*}=0$ is globally asymptotically stable. 
If $\delta<\delta^{*}$, the subsystem (10) has a trivial fixed point $u_{1}^{*}=$ 0 and a positive fixed point

$$
u_{2}^{*}=\frac{r_{1}\left(1-\delta-\exp \left(-r_{1} T\right)\right)}{d_{1}\left(1-\exp \left(-r_{1} T\right)\right)} \text {, }
$$

Moreover,

$$
\left|\frac{d F}{d u}\right|_{u=u_{1}^{*}}=\frac{1-\delta}{e^{-r_{1} T}}>1
$$

So the trivial periodic solution $u_{1}^{*}=0$ is not stable. $u_{2}^{*}$.

Now we consider the stability of the positive fixed point

In fact, if we substitute

$$
x\left(n T^{+}\right)=u_{2}^{*}=\frac{r_{1}\left(1-\delta-\exp \left(-r_{1} T\right)\right)}{d_{1}\left(1-\exp \left(-r_{1} T\right)\right)},
$$

into (11), then we can get

$$
\begin{gathered}
x^{*}(t)=\frac{r_{1}\left(1-\delta-\exp \left(-r_{1} T\right)\right)}{d_{1}\left(1-\delta-\exp \left(-r_{1} T\right)\right)+\delta \exp \left(-r_{1}(t-n T)\right)}, \\
x^{*}\left(n T^{+}\right)=x^{*}\left(0^{+}\right)=\frac{r_{1}\left(1-\delta-\exp \left(-r_{1} T\right)\right)}{d_{1}\left(1-\exp \left(-r_{1} T\right)\right)}, \quad n \in N^{*} .
\end{gathered}
$$

which is a positive periodic solution of system (10).

In the following we will show that the positive periodic solution is globally asymptotically stable.

In order to do this, we take the transformation $x(t)=$ $1 / u(t)$ for system (10), and then the following linear nonhomogeneous impulsive equation is obtained:

$$
\begin{gathered}
\frac{d u}{d t}=d_{1}-r_{1} u(t), \quad t \neq n T, \\
u\left(t^{+}\right)=\frac{1}{1-\delta} u(t), \quad t=n T, n \in N^{*} .
\end{gathered}
$$

Thus, $x(t)=x\left(t, x_{0}\right)$ is the solution of system (10) with initial condition $x\left(0^{+}\right)=x_{0}$ if $u(t)=u\left(t, x_{0}\right)$ is the solution of system (20) with initial condition $u\left(0^{+}\right)=u_{0}=1 / u_{0}$.

Let

$$
n(t, s)=\prod_{s \leq n T<t} \frac{1}{1-\delta} e^{-r_{1}(t-s)}
$$

By the Cauchy matrix of the respective homogeneous equation, we have that

$$
u(t)=n(t, 0) u\left(0^{+}\right)+r_{1} \int_{0}^{t} n(t, s) d s
$$

is the solution of system (20).

Thus,

$$
\left|u(t)-u^{*}(t)\right|=n(t, 0)\left|u\left(0^{+}\right)-u^{*}\left(0^{+}\right)\right| .
$$

On the other hand, when $\delta<\delta^{*}$,

$$
n(t, 0)=\prod_{0 \leq n T<t} \frac{1}{1-\delta} e^{-r_{1} t} \leq\left(\frac{e^{-r_{1} t}}{1-\delta}\right)^{n}, \quad t \in(n T,(n+1) T],
$$

which leads to

$$
\lim _{t \rightarrow \infty} n(t, 0)=\lim _{t \rightarrow \infty}\left(\frac{e^{-r_{1} t}}{1-\delta}\right)^{n}=0 .
$$

Thus,

$$
\begin{aligned}
\mid x & (t)-x^{*}(t) \mid \\
& =\left|\frac{1}{u(t)}-\frac{1}{u^{*}(t)}\right| \\
& =\frac{n(t, 0)\left|u\left(0^{+}\right)-u^{*}\left(0^{+}\right)\right|}{u(t) u^{*}(t)} \longrightarrow 0, \text { as } t \longrightarrow \infty .
\end{aligned}
$$

That is, the positive periodic solution,

$$
\begin{array}{r}
x^{*}(t)=\frac{r_{1}\left(1-\delta-\exp \left(-r_{1} T\right)\right)}{d_{1}\left(1-\delta-\exp \left(-r_{1} T\right)\right)+\delta \exp \left(-r_{1}(t-n T)\right)}, \\
t \in(n T,(n+1) T],
\end{array}
$$

is globally asymptotically stable.

\section{Main Results}

Theorem 6. If $e_{2} \leq e_{1} e_{3}$, then for each solution $X(t)=$ $(x(t), y(t), z(t))$ of system (2), one has

$$
\begin{gathered}
x(t) \leq M_{1} \triangleq \frac{\left(m+r_{1}\right)^{2}}{4 m d_{1}}+\frac{\left(m-r_{2}\right)^{2}}{4 m e_{1} d_{2}}, \\
y(t) \leq M_{2} \triangleq \frac{e_{1}\left(m+r_{1}\right)^{2}}{4 m d_{1}}+\frac{\left(m-r_{2}\right)^{2}}{4 m d_{2}}, \\
z(t) \leq M_{3} \triangleq \frac{e_{1} e_{3}\left(m+r_{1}\right)^{2}}{4 m d_{1}}+e_{3} \frac{\left(m-r_{2}\right)^{2}}{4 m d_{2}},
\end{gathered}
$$

when $t$ is large enough.

Proof. Let $X(t)=(x(t), y(t), z(t))$ be any solution of system (2) with initial condition (3), and we define

$$
W(t)=e_{1} e_{3} x(t)+e_{3} y(t)+z(t+\tau)
$$

Then,

$$
\begin{aligned}
\left.\frac{d W}{d t}\right|_{(2)}= & e_{1} e_{3} x(t)\left(r_{1}-d_{1} x(t)\right)-\frac{a_{2} e_{1} e_{3} x(t) z(t)}{b_{2}+x(t)} \\
& -r_{2} e_{3} y(t)-d_{2} e_{3} y^{2}(t)+\frac{a_{2} e_{2} x(t) z(t)}{b_{2}+x(t)} \\
& -m z(t+\tau),
\end{aligned}
$$


which yields

$$
\begin{aligned}
&\left.\frac{d W}{d t}\right|_{(2)}+m W(t) \\
&=e_{1} e_{3} x(t)\left(\left(r_{1}+m\right)-d_{1} x(t)\right)+e_{3}\left(m-r_{2}\right) y(t) \\
& \quad-e_{3} d_{2} y^{2}(t)+\frac{a_{2}\left(e_{2}-e_{1} e_{3}\right) x(t) z(t)}{b_{2}+x(t)} \\
& \leq e_{1} e_{3}\left(\left(r_{1}+m\right) x(t)-d_{1} x^{2}(t)\right) \\
& \quad+e_{3}\left(\left(m-r_{2}\right) y(t)-d_{2} y^{2}(t)\right) \\
& \leq \frac{e_{1} e_{3}\left(m+r_{1}\right)^{2}}{4 d_{1}}+\frac{e_{3}\left(m-r_{2}\right)^{2}}{4 d_{2}} \triangleq L .
\end{aligned}
$$

On the other hand, by a simple calculation

$$
\begin{aligned}
W\left(t^{+}\right) & =(1-\delta) e_{1} e_{3} x(t)+e_{3} y(t)+z(t+\tau) \\
& \leq e_{1} e_{3} x(t)+e_{3} y(t)+z(t+\tau) \\
& =W(t) .
\end{aligned}
$$

Therefore,

$$
\begin{gathered}
\left.\frac{d W}{d t}\right|_{(2)} \leq-m W(t)+L, \quad t \neq n T, \\
W\left(t^{+}\right) \leq W(t), \quad t=n T, n \in N^{*} .
\end{gathered}
$$

By Lemma 2.2 in [14] we have

$$
\begin{aligned}
W(t) & =W\left(0^{+}\right) e^{-m t}+\int_{0}^{t} L e^{-m(t-s)} d s \\
& \leq W\left(0^{+}\right) e^{-m t}+\frac{L}{m} \longrightarrow \frac{L}{m}, \quad \text { as } t \longrightarrow \infty,
\end{aligned}
$$

which leads to

$$
\begin{gathered}
x(t) \leq \frac{L}{m e_{1} e_{3}}=M_{1}, \quad y(t) \leq \frac{L}{m e_{3}}=M_{2}, \\
z(t) \leq \frac{L}{m}=M_{3} .
\end{gathered}
$$

This completes the proof of this theorem.

Now we begin to study the global attractivity of predatorseradication periodic solution $\left(x^{*}(t), 0,0\right)$ of system (2), which is the circumstance when both of the predator individuals are entirely absent from the system ultimately; that is, $y(t)=0$ and $z(t)=0$.

Theorem 7. If system (2) satisfies $\delta<\delta^{*}$ and the following condition (H1):

$$
\begin{aligned}
T<T_{1}^{*} \triangleq \min \left\{\frac{1}{r_{1}} \ln \frac{r_{1} e_{1} a_{1}-r_{1} r_{2}-d_{1} b_{1} r_{2}(1-\delta)}{(1-\delta)\left(r_{1} e_{1} a_{1}-r_{1} r_{2}-d_{1} b_{1} r_{2}\right)} ;\right. \\
\left.\frac{1}{r_{1}} \ln \frac{r_{1} e_{2} a_{2}-m r_{1}-m d_{1} b_{1}(1-\delta)}{(1-\delta)\left(r_{1} e_{2} a_{2}-m r_{1}-m d_{1} b_{1}\right)}\right\},
\end{aligned}
$$

then the predators-eradication periodic solution $\left(x^{*}(t), 0,0\right)$ of system (2) is globally attractive.

Proof. By the first equation and the impulsive effect, we have

$$
\begin{gathered}
\frac{d x}{d t} \leq x(t)\left(r_{1}-d_{1} x(t)\right), \quad t \neq n T, n \in N^{*}, \\
x\left(t^{+}\right)=(1-\delta) x(t), \quad t=n T, n \in N^{*}, \\
x\left(0^{+}\right)=x_{0},
\end{gathered}
$$

whose comparison system is (10).

Then, by comparison theorem (Lemma 3) of impulsive differential equations, there exists an arbitrarily small positive $\varepsilon>0$ such that

$$
x(t)<x^{*}(t)+\varepsilon,
$$

when $t$ is large enough.

This yields

$$
\lim _{t \rightarrow \infty} \sup x(t) \leq \frac{r_{1}\left(1-\delta-\exp \left(-r_{1} T\right)\right)}{d_{1}\left(1-\delta-\exp \left(-r_{1} T\right)+\delta \exp \left(-r_{1} T\right)\right)} .
$$

Hence, there exists a positive integer $n_{1} \in N$ and arbitrarily small positive $\varepsilon_{1}>0$ such that

$$
x(t) \leq \frac{r_{1}\left(1-\delta-\exp \left(-r_{1} T\right)\right)}{d_{1}\left(1-\delta-\exp \left(-r_{1} T\right)+\delta \exp \left(-r_{1} T\right)\right)}+\varepsilon_{1} \triangleq \eta_{1},
$$

for all $t \geq n_{1} T$.

On the other hand, since condition ( $\mathrm{H} 1)$ holds, then $e_{1} a_{1} \eta_{1} /\left(b_{1}+\eta_{1}\right)<r_{2}$ for above $\varepsilon_{1}>0$ small enough.

At the moment, from the second equation of the system (2) we have

$$
\begin{aligned}
\frac{d y}{d t} & \leq \frac{e_{1} a_{1} \eta_{1}}{b_{1}+\eta_{1}} y(t)-r_{2} y(t)-d_{2} y^{2}(t) \\
& =\left(\frac{e_{1} a_{1} \eta_{1}}{b_{1}+\eta_{1}}-r_{2}\right) y(t)-d_{2} y^{2}(t)<0 .
\end{aligned}
$$

Then,

$$
\lim _{t \rightarrow \infty} y(t)=0 \text {. }
$$

Then there exist $T_{1}>0$ and $\varepsilon_{2}>0$ small enough, such that

$$
0<y(t)<\varepsilon_{2}, \quad \forall t>T_{1},
$$

and it follows from the last equation of system (2) that

$$
\frac{d z}{d t} \leq\left(\frac{e_{2} a_{2} \eta_{1}}{b_{2}+\eta_{1}}+\frac{e_{3} a_{3} \varepsilon_{2}}{b_{3}+\varepsilon_{2}}\right) z(t-\tau)-m z(t)
$$

when $t>\max \left\{T_{1}, n_{1} T\right\}+\tau$. 
For above arbitrarily small positive $\varepsilon_{1}, \varepsilon_{2}$ small enough, since condition ( $\mathrm{H} 1)$ holds, then

$$
\frac{e_{2} a_{2} \eta_{1}}{b_{2}+\eta_{1}}+\frac{e_{3} a_{3} \varepsilon_{2}}{b_{3}+\varepsilon_{2}}<m
$$

By Lemma 4, we have

$$
\lim _{t \rightarrow \infty} z(t)=0 .
$$

Then for above $\varepsilon_{2}>0$ small enough, there exists a $T_{2}>T_{1}$ such that

$$
0<z(t)<\varepsilon_{2}, \quad \forall t>T_{2} .
$$

On the other hand, combining the first equation of system (2) with (43) and (47), we have

$$
\begin{aligned}
\frac{d x}{d t} & \geq x(t)\left(r_{1}-d_{1} x(t)\right)-\left(\frac{a_{1} \varepsilon_{2}}{b_{1}}+\frac{a_{2} \varepsilon_{2}}{b_{2}}\right) \\
& =x(t)\left(\gamma_{1}-d_{1} x(t)\right),
\end{aligned}
$$

for $t>T_{2}$, where

$$
\gamma_{1} \triangleq r_{1}-\left(\frac{a_{1}}{b_{1}}+\frac{a_{2}}{b_{2}}\right) \varepsilon_{2} .
$$

Note that the corresponding comparison system of (48) is

$$
\begin{gathered}
\frac{d u}{d t}=u(t)\left(\gamma_{1}-d_{1} u(t)\right), \quad t \neq n T, \\
u\left(t^{+}\right)=(1-\delta) u(t), \quad t=n T, n \in N^{*}, \\
u\left(0^{+}\right)=x_{0} .
\end{gathered}
$$

By Lemma 5 , if $\delta<\delta^{*}$, system (50) also has the following positive periodic solution:

$$
u^{*}(t)=\frac{\gamma_{1}\left(1-\delta-\exp \left(-\gamma_{1} T\right)\right)}{d_{1}\left(1-\delta-\exp \left(-\gamma_{1} T\right)+\delta \exp \left(-\gamma_{1}(t-n T)\right)\right)},
$$

which is globally asymptotically stable.

Thus, by Lemma 3 again we have

$$
x(t)>u^{*}(t)-\varepsilon,
$$

for above arbitrarily small $\varepsilon>0$ as $t$ is large enough.

Let $\varepsilon_{2} \rightarrow 0$, and then

$$
\gamma_{1} \triangleq r_{1}-\left(\frac{a_{1}}{b_{1}}+\frac{a_{2}}{b_{2}}\right) \varepsilon_{2} \longrightarrow r_{1},
$$

that is, $u^{*}(t) \rightarrow x^{*}(t)$.

At this time, it follows from (38) and (52) that

$$
x^{*}(t)-\varepsilon<x(t)<x^{*}(t)+\varepsilon .
$$

Thus, for $t>T_{2}$ large enough, we have

$$
\lim _{t \rightarrow \infty} x(t)=x^{*}(t) \text {. }
$$

Combined with (42), (46), and (55), we have proved that the predators-eradication periodic solution $\left(x^{*}(t), 0,0\right)$ of system (2) is globally attractive.
Corollary 8. If system (2) satisfies $\delta<\delta^{*}$ and

$$
\begin{aligned}
& \delta>\Delta^{*}=\max \left\{\frac{\left(r_{1} e_{1} a_{1}-r_{1} r_{2}-r_{2} b_{1} d_{1}\right) \delta^{*}}{r_{1} e_{1} a_{1}-r_{1} r_{2}-r_{2} b_{1} d_{1} \delta^{*}},\right. \\
& \left.\frac{\left(r_{1} e_{2} a_{2}-m r_{1}-m b_{1} d_{1}\right) \delta^{*}}{r_{1} e_{2} a_{2}-m r_{1}-m b_{1} d_{1} \delta^{*}}\right\} \text {, }
\end{aligned}
$$

then the predators-eradication periodic solution $\left(x^{*}(t), 0,0\right)$ of system (2) is globally attractive.

In fact, if the conditions of Corollary 8 hold, then

$$
\begin{gathered}
\delta>\frac{\left(r_{1} e_{2} a_{2}-m r_{1}-m b_{1} d_{1}\right) \delta^{*}}{r_{1} e_{2} a_{2}-m r_{1}-m b_{1} d_{1} \delta^{*}}, \\
\delta>\frac{\left(r_{1} e_{1} a_{1}-r_{1} r_{2}-r_{2} b_{1} d_{1}\right) \delta^{*}}{r_{1} e_{1} a_{1}-r_{1} r_{2}-r_{2} b_{1} d_{1} \delta^{*}} .
\end{gathered}
$$

It follows from (57) that

$$
\left(r_{1} e_{2} a_{2}-m r_{1}-m b_{1} d_{1}\right) \delta^{*}+m d_{1} b_{1} \delta \delta^{*}<\left(r_{1} e_{2} a_{2}-m r_{1}\right) \delta,
$$

which yields

$$
\delta^{*}<\frac{\left(r_{1} e_{2} a_{2}-m r_{1}\right) \delta}{r_{1} e_{2} a_{2}-m r_{1}-m b_{1} d_{1}(1-\delta)} .
$$

Note that

$$
\delta^{*}=1-e^{-r_{1} T},
$$

and then

$$
e^{-r_{1} T}>\frac{(1-\delta)\left(r_{1} e_{2} a_{2}-m r_{1}-m b_{1} d_{1}\right)}{r_{1} e_{2} a_{2}-m r_{1}-m b_{1} d_{1}(1-\delta)} .
$$

That is,

$$
T<\frac{1}{r_{1}} \ln \frac{r_{1} e_{1} a_{1}-r_{1} r_{2}-d_{1} b_{1} r_{2}(1-\delta)}{(1-\delta)\left(r_{1} e_{1} a_{1}-r_{1} r_{2}-d_{1} b_{1} r_{2}\right)} .
$$

In the same way, from (58), we have another inequality:

$$
T<\frac{1}{r_{1}} \ln \frac{r_{1} e_{2} a_{2}-m r_{1}-m d_{1} b_{1}(1-\delta)}{(1-\delta)\left(r_{1} e_{2} a_{2}-m r_{1}-m d_{1} b_{1}\right)} .
$$

Therefore, all the conditions of Theorem 7 hold, and then the predators-eradication periodic solution $\left(x^{*}(t), 0,0\right)$ of system (2) is globally attractive.

Remark 9. From Theorem 7 and its sufficient condition Corollary 8 , if $\delta<\delta^{*}, T<T_{1}^{*}$, or $\Delta^{*}<\delta<\delta^{*}$, then the natural enemies (both of the predators' population) in the model are extinct while the pest population is still not controlled when the pest population is poisoned exclusively. From the viewpoint of ecosystem and protecting the variety of the rare species, we only need to control the pest population under a certain threshold level and should not eradicate the enemy population. That is, the pest population and the enemy population can coexist when the pest cannot cause immense economic losses, so it is more important to consider the uniform persistence for the system. 
Theorem 10. If system (2) satisfies $e_{2} \leq e_{1} e_{3}, \delta<\bar{\delta}$, and the following condition (H2):

$$
\begin{aligned}
& T>T_{2}^{*} \\
& \triangleq \max \left\{\frac{1}{\theta_{1}} \ln \frac{\theta_{1}\left(e_{2} a_{2}-m\right)-m d_{1} b_{2}}{\theta_{1}(1-\delta)\left(e_{2} a_{2}-m\right)-m d_{1} b_{2}} ;\right. \\
& \frac{1}{\theta_{2}} \ln \left(\left(\theta_{2}\left(b_{3} e_{1} a_{1}-b_{3} r_{2}-a_{3} M_{3}\right)\right.\right. \\
& \left.-b_{1} d_{1}\left(b_{3} r_{2}+a_{3} M_{3}\right)\right) \\
& \times\left(\theta_{2}(1-\delta)\left(b_{3} e_{1} a_{1}-b_{3} r_{2}-a_{3} M_{3}\right)\right. \\
& \left.\left.\left.-b_{1} d_{1}\left(b_{3} r_{2}+a_{3} M_{3}\right)\right)^{-1}\right)\right\},
\end{aligned}
$$

where $\bar{\delta}=1-e^{-\gamma^{*} T}, \gamma^{*}=r_{1}-\left(a_{1} M_{2} / b_{1}+a_{2} M_{3} / b_{2}\right), \theta_{1}=r_{1}-$ $a_{1} M_{2} / b_{1}$, and $\theta_{2}=r_{2}-a_{2} M_{3} / b_{2}$, then system (2) is permanent.

Proof. From Theorem 6, we have obtained the upper bound of each solution $X(t)=(x(t), y(t), z(t))$ of the system (2) with $t$ large enough. Thus, we only need to search for the lower bound of the solution in the following.

In fact, from the first equation of system (7), we have

$$
\begin{aligned}
\frac{d x}{d t} & \geq x(t)\left(r_{1}-d_{1} x(t)\right)-\left(\frac{a_{1} M_{2}}{b_{1}}+\frac{a_{2} M_{3}}{b_{2}}\right) x(t) \\
& =x(t)\left(\gamma^{*}-d_{1} x(t)\right) .
\end{aligned}
$$

By the comparison theorem (Lemma 3) we have $x(t) \geq$ $v_{1}(t)$ and $v_{1}(t) \rightarrow \widetilde{v_{1}}(t)$ as $t \rightarrow \infty$, where $v_{1}(t)$ is the unique and globally stable positive periodic solution of

$$
\begin{gathered}
\frac{d v_{1}}{d t}=v_{1}(t)\left(\gamma^{*}-d_{1} v_{1}(t)\right), \quad t \neq n T, n \in N^{*} \\
v_{1}\left(t^{+}\right)=(1-\delta) v_{1}(t), \quad t=n T, n \in N^{*}, \\
v_{1}\left(0^{+}\right)=x_{0}>0, \\
\widetilde{v_{1}}(t)=\frac{\gamma^{*}\left(1-\delta-\exp \left(-\gamma^{*} T\right)\right)}{d_{1}\left(1-\delta-\exp \left(-\gamma^{*} T\right)+\delta \exp \left(-\gamma^{*}(t-n T)\right)\right)} .
\end{gathered}
$$

Therefore, for sufficiently large $t$, there exists a $\varepsilon_{3}>0$ small enough such that

$$
x(t) \geq \widetilde{v_{1}}(t)-\varepsilon_{3}>\frac{\gamma^{*}\left(1-\delta-\exp \left(-\gamma^{*} T\right)\right)}{d_{1}\left(1-\exp \left(-\gamma^{*} T\right)\right)}-\varepsilon_{3} \triangleq m_{1} .
$$

In the following, we will show that there exist two positive constants $\bar{m}_{2}$ and $\bar{m}_{3}$, such that $y(t) \geq \bar{m}_{2}$ and $z(t) \geq \bar{m}_{3}$ for any $t$ large enough.

Step 1. We begin to find an $\bar{m}_{2}>0$ such that $y(t) \geq \bar{m}_{2}$ for any $t$ large enough.
In order to achieve this goal, firstly we claim that the inequality $y(t)<m_{2}$ cannot hold for all $t \geq t_{1}$.

Otherwise, if $y(t)<m_{2}$ for all $t \geq t_{1}$, then from the first equation of (2),

$$
\begin{aligned}
\frac{d x}{d t} & \geq x(t)\left(r_{1}-d_{1} x(t)\right)-\left(\frac{a_{1} m_{2}}{b_{1}}+\frac{a_{2} M_{3}}{b_{2}}\right) x(t) \\
& =x(t)\left(\gamma_{2}-d_{1} x(t)\right),
\end{aligned}
$$

where

$$
\gamma_{2}=r_{1}-\left(\frac{a_{1} m_{2}}{b_{1}}+\frac{a_{2} M_{3}}{b_{2}}\right) .
$$

Therefore, there exists a $\varepsilon_{4}>0$ small enough and a $T_{3} \geq$ $t_{1}$, such that, for $t \geq T_{3}$,

$$
x(t) \geq \widetilde{v_{2}}(t)-\varepsilon_{4}>\frac{\gamma_{2}\left(1-\delta-\exp \left(-\gamma_{2} T\right)\right)}{d_{1}\left(1-\exp \left(-\gamma_{2} T\right)\right)}-\varepsilon_{4} \triangleq \eta_{2},
$$

where

$$
\widetilde{v_{2}}(t)=\frac{\gamma_{2}\left(1-\delta-\exp \left(-\gamma_{2} T\right)\right)}{d_{1}\left(1-\delta-\exp \left(-\gamma_{2} T\right)+\delta \exp \left(-\gamma_{2}(t-n T)\right)\right)} .
$$

When condition (H2) holds, we can choose $m_{2}, \varepsilon_{4}>0$ small enough such that

$$
\sigma_{1}=\int_{n T}^{(n+1) T}\left(\frac{e_{1} a_{1} \eta_{2}}{b_{1}+\eta_{2}}-\frac{a_{3} M_{3}}{b_{3}}-r_{2}-d_{2} m_{2}\right) d t>0 .
$$

Then at this time, from the second equation of system (2),

$$
\frac{d y}{d t} \geq\left(\frac{e_{1} a_{1} \eta_{2}}{b_{1}+\eta_{2}}-\frac{a_{3} M_{3}}{b_{3}}-r_{2}-d_{2} m_{2}\right) y(t) .
$$

Let $n_{1} \in N^{*}, N_{1} T \geq T_{3}$ and integrate (74) on $(n T,(n+$ 1)T], and we can get

$$
\begin{aligned}
& y((n+1) T) \\
& \geq y\left(n T^{+}\right) \\
& \quad \times \exp \left(\int_{n T}^{(n+1) T}\left(\frac{e_{1} a_{1} \eta_{2}}{b_{1}+\eta_{2}}-\frac{a_{3} M_{3}}{b_{3}}-r_{2}-d_{2} m_{2}\right) d t\right) \\
& =y(n T) \exp \left(\sigma_{1}\right) .
\end{aligned}
$$

Then $y\left(\left(N_{1}+k\right) T\right) \geq y\left(N_{1} T\right) \exp \left(k \sigma_{1}\right) \rightarrow+\infty$ as $k \rightarrow$ $+\infty$, which is contradicted with $y(t) \leq M_{2}$.

Hence, there exists a $t_{2} \geq t_{1}>0$, such that $y\left(t_{2}\right) \geq m_{2}$. If $y(t) \geq m_{2}$ for all $t \geq t_{2}$, then our aim is obtained.

Otherwise, if $y(t)$ is oscillatory around $m_{2}$, let

$$
\bar{m}_{2}=\min \left\{\frac{m_{2}}{2}, m_{2} \exp \left(-\left(\frac{a_{3} M_{3}}{b_{3}}+r_{2}+d_{2} m_{2}\right) T\right)\right\} .
$$


And we assume that there exists two positive constants $\bar{t}_{1}\left(>t_{2}\right)$ and $w_{1}>0$ such that

$$
\begin{array}{r}
y\left(\bar{t}_{1}\right)=y\left(\bar{t}_{1}+w_{1}\right)=m_{2}, \\
y(t)<m_{2} \text { for } \bar{t}_{1}<t<\bar{t}_{1}+w_{1} .
\end{array}
$$

Since $y(t)$ is continuous, bounded, and not affected by impulses, we conclude that $y(t)$ is uniformly continuous; then exists a $T_{4}>0$ (with $0<T_{4}<T$ and $T_{4}$ is independent of the choice of $\left.\bar{t}_{1}\right)$ such that $y(t) \geq m_{2} / 2$ for all $\bar{t}_{1}<t<\bar{t}_{1}+T_{4}$.

If $w_{1} \leq T_{4}$, then $y(t) \geq m_{2} / 2 \geq \bar{m}_{2}$ for all $\bar{t}_{1}<t<\bar{t}_{1}+w_{1}$.

If $T_{4}<w_{1} \leq T$, then, from the second equation of (2),

$$
\frac{d y}{d t} \geq-\left(\frac{a_{3} M_{3}}{b_{3}}+r_{2}+d_{2} m_{2}\right) y(t), \quad \text { for } \bar{t}_{1}<t<\bar{t}_{1}+w_{1} \text {. }
$$

Integrate (78) on $\left[\bar{t}_{1}, t\right](\bar{t} \leq t \leq T)$, and we have

$$
\begin{aligned}
y(t) & \geq y\left(\bar{t}_{1}\right) \exp \left(-\left(\frac{a_{3} M_{3}}{b_{3}}+r_{2}+d_{2} m_{2}\right)\left(t-\bar{t}_{1}\right)\right) \\
& \geq m_{2} \exp \left(-\left(\frac{a_{3} M_{3}}{b_{3}}+r_{2}+d_{2} m_{2}\right) T\right) \geq \bar{m}_{2} .
\end{aligned}
$$

If $T_{4}<T<w_{1}$, from the second equation of (2), we can also obtain

$$
y(t) \geq \bar{m}_{2} \quad \forall \bar{t}_{1} \leq t \leq \bar{t}_{1}+T .
$$

Proceeding exactly as above analysis, we can conclude that $y(t) \geq \bar{m}_{2}$, for $\bar{t}_{1}+T \leq t \leq \bar{t}_{1}+w_{1}$.

Thus, no matter which case we have $y(t) \geq \bar{m}_{2}$ for all $\bar{t}_{1} \leq$ $t \leq \bar{t}_{1}+w_{1}$, since the interval $\left[\bar{t}_{1}, \bar{t}_{1}+w_{1}\right]$ is arbitrarily chosen, then there exist $\bar{m}_{2}>0$, such that $y(t) \geq \bar{m}_{2}$ for $t$ is large enough.

Step 2. Now we try to find an $\bar{m}_{3}>0$ such that $z(t) \geq \bar{m}_{3}$ for all $t$ is large enough.

In the same method, we claim that the inequality $z(t)<$ $m_{3}$ cannot hold for all $t>t_{3}$.

Otherwise, if there exists a $t_{3}>0$ such that $z(t)<m_{3}$ for all $t \geq t_{3}+\tau$, then by the first equation of (2),

$$
\begin{aligned}
\frac{d x}{d t} & \geq x(t)\left(r_{1}-d_{1} x(t)\right)-\left(\frac{a_{1} M_{2}}{b_{1}}+\frac{a_{2} m_{3}}{b_{2}}\right) x(t) \\
& =x(t)\left(\gamma_{3}-d_{1} x(t)\right),
\end{aligned}
$$

where

$$
\gamma_{3}=r_{1}-\left(\frac{a_{1} M_{2}}{b_{1}}+\frac{a_{2} m_{3}}{b_{2}}\right) .
$$

Therefore, there exists a $\varepsilon_{5}>0$ small enough and a $t_{4} \geq$ $t_{3}+\tau$, such that for $t \geq t_{4}$,

$$
x(t) \geq \widetilde{v_{3}}(t)-\varepsilon_{5}>\frac{\gamma_{3}\left(1-\delta-\exp \left(-\gamma_{3} T\right)\right)}{d_{1}\left(1-\exp \left(-\gamma_{3} T\right)\right)}-\varepsilon_{5} \triangleq \eta_{3},
$$

where

$$
\widetilde{v_{3}}(t)=\frac{\gamma_{3}\left(1-\delta-\exp \left(-\gamma_{3} T\right)\right)}{d_{1}\left(1-\delta-\exp \left(-\gamma_{3} T\right)+\delta \exp \left(-\gamma_{3}(t-n T)\right)\right)} .
$$

Now we define a Liapunov functional

$V(t)=z(t)+\int_{t-\tau}^{t} \frac{e_{2} a_{2} x(s) z(s)}{b_{2}+x(s)} d s+\int_{t-\tau}^{t} \frac{e_{3} a_{3} y(s) z(s)}{b_{3}+y(s)} d s$,

and then

$$
\begin{aligned}
\left.\frac{d V}{d t}\right|_{(2)} & =\left(\frac{e_{2} a_{2} x(t)}{b_{2}+x(t)}+\frac{e_{3} a_{3} y(t)}{b_{3}+y(t)}-m\right) z(t) \\
& >\left(\frac{e_{2} a_{2} \eta_{3}}{b_{2}+\eta_{3}}-m\right) z(t) .
\end{aligned}
$$

When the condition (H2) holds, we can choose $\varepsilon_{5}>0$ small enough such that

$$
\begin{aligned}
& \frac{e_{2} a_{2} \eta_{3}}{b_{2}+\eta_{3}}-m \\
& =\frac{e_{2} a_{2}\left(\gamma_{3}\left(1-\delta-\exp \left(-\gamma_{3} T\right)\right) / d_{1}\left(1-\exp \left(-\gamma_{3} T\right)\right)-\varepsilon_{5}\right)}{b_{2}+\gamma_{3}\left(1-\delta-\exp \left(-\gamma_{3} T\right)\right) / d_{1}\left(1-\exp \left(-\gamma_{3} T\right)\right)-\varepsilon_{5}} \\
& \quad-m>0 .
\end{aligned}
$$

Let $z^{L}=\min _{t \in\left[t_{4}, t_{4}+\tau\right]} z(t)$, and we claim that

$$
z(t) \geq z^{L}, \quad \forall t \geq t_{4} .
$$

Otherwise, if there exists a nonnegative constant $t_{5} \geq t_{4}+$ $\tau$ such that

$$
\begin{gathered}
z\left(t_{5}\right)=z^{L}, \quad z(t) \geq z^{L} \quad \text { for } t \in\left[t_{4}, t_{5}\right], \\
\dot{z}\left(t_{5}\right) \leq 0 .
\end{gathered}
$$

When $t>t_{5} \geq t_{4}+\tau$, from the last equation of (2), we have

$$
\dot{z}(t) \geq\left(\frac{e_{2} a_{2} \eta_{3}}{b_{2}+\eta_{3}}-m\right) z(t) .
$$

Thus,

$$
\dot{z}\left(t_{5}\right) \geq\left(\frac{e_{2} a_{2} \eta_{3}}{b_{2}+\eta_{3}}-m\right) z\left(t_{5}\right)=\left(\frac{e_{2} a_{2} \eta_{3}}{b_{2}+\eta_{3}}-m\right) z^{L}
$$

which is a contradiction.

Therefore,

$$
\begin{aligned}
\left.\frac{d V}{d t}\right|_{(2)} & =\left(\frac{e_{2} a_{2} x(t)}{b_{2}+x(t)}+\frac{e_{3} a_{3} y(t)}{b_{3}+y(t)}-m\right) z(t) \\
& >\left(\frac{e_{2} a_{2} \eta_{3}}{b_{2}+\eta_{3}}-m\right) z^{L}>0, \quad \forall t>t_{5} .
\end{aligned}
$$


which implies $V(t) \rightarrow+\infty$, as $t \rightarrow+\infty$, and this is contradicted with

$$
V(t) \leq M_{3}+\frac{e_{2} a_{2} M_{1} M_{3} \tau}{b_{2}+M_{1}}+\frac{e_{3} a_{3} M_{2} M_{3} \tau}{b_{3}+M_{2}} .
$$

Therefore, $z(t)<m_{3}$ cannot hold for all $t \geq t_{3}$, and there are two cases as follows.

If $z(t) \geq m_{3}$ for all $t \geq t_{3}$, then our aim is obtained.

Otherwise, if $z(t)$ is oscillatory around $m_{3}$, when $t$ is sufficiently large, let

$$
\bar{m}_{3}=\min \left\{\frac{m_{3}}{2}, m_{3} \exp (-m \tau)\right\},
$$

then we can show that $z(t) \geq \bar{m}_{3}$ as $t$ is large enough.

In fact, suppose there exist two positive constants $\bar{t}_{2}>$ $0, w_{2}>0$, such that

$$
\begin{gathered}
z\left(\bar{t}_{2}\right)=z\left(\bar{t}_{2}+w_{2}\right)=m_{3}, \\
z(t)<m_{3} \text { for } \bar{t}_{2}<t<\bar{t}_{2}+w_{2} .
\end{gathered}
$$

Since $z(t)$ is continuous, bounded, and not affected by impulses, we conclude that $z(t)$ is uniformly continuous; then exists a $T_{5}>0$ (with $0<T_{5}<\tau$ and $T_{5}$ is independent of the choice of $\bar{t}_{2}$ ) such that $z(t) \geq m_{3} / 2$ for all $\bar{t}_{2}<t<\bar{t}_{2}+T_{5}$.

If $w_{2} \leq T_{5}$, then $z(t) \geq m_{3} / 2 \geq \bar{m}_{3}$ for all $\bar{t}_{2}<t<\bar{t}_{2}+w_{2}$.

If $T_{5}<w_{2} \leq \tau$, then from the last equation of (2),

$$
\frac{d z}{d t} \geq-m z(t), \quad \text { for } \bar{t}_{2} \leq t \leq \bar{t}_{2}+w_{2} \leq \bar{t}_{2}+\tau .
$$

Integrate (96) on $\left[\bar{t}_{2}, t\right](\bar{t} \leq t \leq \tau)$, and we have

$$
\begin{array}{r}
z(t) \geq z\left(\bar{t}_{2}\right) \exp \left(-m\left(t-\bar{t}_{2}\right)\right) \geq m_{3} \exp (-m \tau), \\
\text { for } \bar{t}_{2} \leq t \leq \bar{t}_{2}+w_{2} .
\end{array}
$$

If $T_{5}<\tau<w_{2}$, from the second equation of system (2), we can also obtain

$$
z(t) \geq m_{3} \exp (-m \tau), \quad \text { for } \bar{t}_{2} \leq t \leq \bar{t}_{2}+\tau .
$$

Proceeding exactly as the proof for above claim (88), we can obtain $z(t) \geq m_{3} \exp (-m \tau)$ for all $t>\bar{t}_{2}$, then $z(t) \geq$ $m_{3} \exp (-m \tau) \geq \bar{m}_{3}$ for $\bar{t}_{2} \leq t \leq \bar{t}_{2}+w_{2}$.

Thus, no matter which case we have $z(t) \geq \bar{m}_{3}$ for all $\bar{t}_{2} \leq$ $t \leq \bar{t}_{2}+w_{2}$, since the interval $\left[\bar{t}_{2}, \bar{t}_{2}+w_{2}\right]$ is arbitrarily chosen, then there exist $\bar{m}_{3}>0$, such that $z(t) \geq \bar{m}_{3}$ for $t$ is large enough.

Set $\Omega=\left\{(x, y, z) \mid m_{1} \leq x(t) \leq M_{1}, m_{2} \leq y(t) \leq\right.$ $\left.M_{2}, m_{3} \leq z(t) \leq M_{3}\right\}$. From above proof, we know that $\Omega$ is the global attractor, and each solution of system (2) will eventually enter and remain in region $\Omega$. According to Definition 2, system (2) is permanent.

In a similar way to the discussion of Corollary 8, we can obtain the following two sufficient conditions for the permanence.
Corollary 11. If system (2) satisfies $e_{2} \leq e_{1} e_{3}$ and

$$
\begin{aligned}
\delta<\min \{1, & \frac{\theta_{1} e_{2} a_{2}-m \theta_{1}-m b_{2} d_{1}}{\theta_{1}\left(e_{2} a_{2}-m\right)}, \\
& \left(\theta_{2} e_{1} a_{1} b_{3}-\theta_{2} e_{3} M_{3}-\theta_{2} b_{2} r_{2}\right. \\
& \left.-b_{1} d_{1} e_{3} M_{3}-b_{1} d_{1} b_{2} r_{2}\right) \\
& \left.\times\left(\theta_{2}\left(e_{1} a_{1} b_{3}-e_{3} M_{3}-b_{2} r_{2}\right)\right)^{-1}\right\} \bar{\delta},
\end{aligned}
$$

where, $\theta_{1}, \theta_{2}$, and $\gamma^{*}, \bar{\delta}$ is the same as Theorem 10 , then system (2) is permanent.

Corollary 12. If system (2) satisfies

$$
\begin{gathered}
R_{2}^{*}=\max \left\{\frac{\delta}{1-e^{-\gamma * T}}, \frac{e_{2}}{e_{1} e_{3}}\right\}<1, \\
R_{*}=\min \left\{\frac{\theta_{1}\left(e_{2} a_{2}-m\right)(\bar{\delta}-\delta)}{m b_{2} d_{1} \bar{\delta}},\right. \\
\left.\frac{\theta_{2}\left(e_{1} a_{1} b_{3}-e_{3} M_{3}-b_{2} r_{2}\right)(\bar{\delta}-\delta)}{b_{1} d_{1}\left(e_{3} M_{3}+b_{2} r_{2}\right) \bar{\delta}}\right\}>1,
\end{gathered}
$$

where $\theta_{1}, \theta_{2}$ and $\gamma^{*}, \bar{\delta}$ is the same as Theorem 10 , then system (2) is permanent.

\section{Numerical Simulations and Discussions}

In this paper, we consider a food-chain prey-predator system with digest delay and impulsive harvest on the prey. Our main aim is to investigate how the impulsive harvest and digest delay affect the dynamical behavior of the system. Especially, we focus on the suitable impulsive period so that we could guarantee that the predators will not be extinct before the prey. Furthermore, we are also concerned when the system will be permanent and how to control the population of the prey (pests) under a certain economic threshold level (ETL).

In the following, we will verify our main results by numerical simulation.

Case 1. If we choose $r_{1}=0.98, r_{2}=0.05, d_{1}=0.001$, $d_{2}=0.01, a_{1}=0.02, a_{2}=0.03, a_{3}=0.01, e_{1}=4, e_{2}=0.8$, $e_{3}=2.131, b_{1}=30, b_{2}=50, b_{3}=1.5, m=0.02, \tau=0.1$, $\delta=0.856$, and $T=2$ with initial conditions $x(0)=5$, $y(0)=3$, and $z(0)=0.5$, it is easy to calculate $\delta^{*}=0.8591$, $T_{1}^{*}=2.008$, and $\delta=0.856<\delta^{*}, T=2<T_{1}^{*}$, which satisfies the condition of Theorem 7. From the time-series diagram of $x(t), y(t)$, and $z(t)$ (see Figures 1(a), 1(b), and 1(c)), we can see that the predators $y(t)$ and $z(t)$ become extinct while the prey population (pests population) is much more than the initial $x(0)=5$, and Figure 1(d) is the phase portrait of this circumstance. This means that when we capture or poison the pests more frequently, natural enemies will become extinct before the pests while the number of pests may increase than before. 


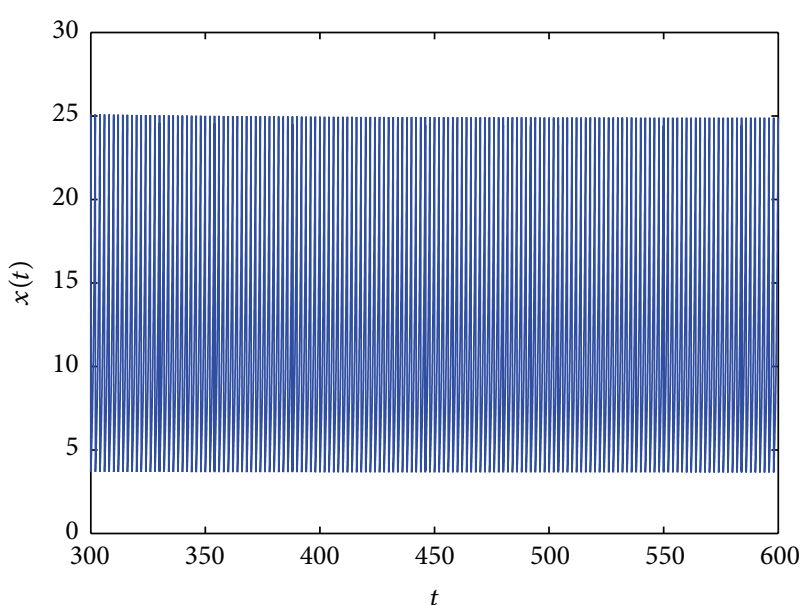

(a)

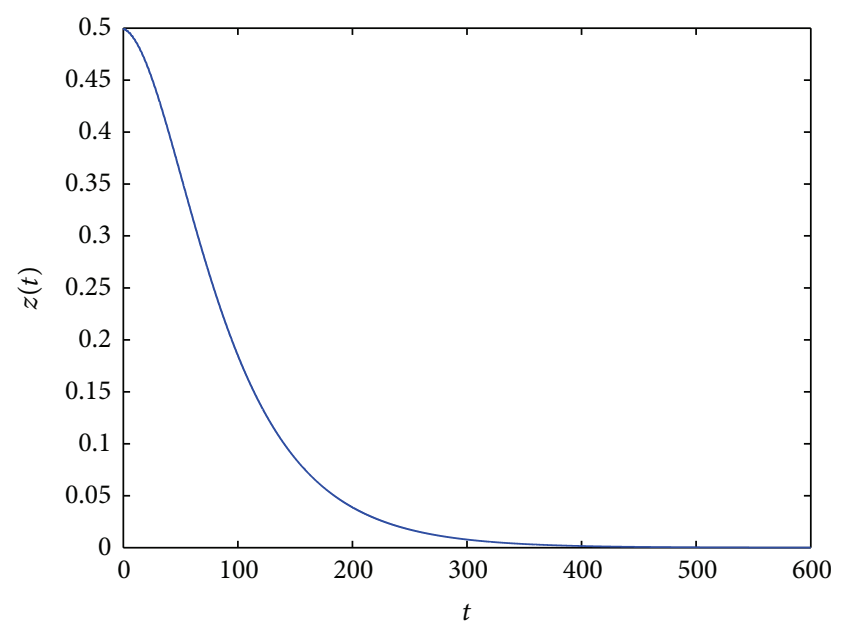

(c)

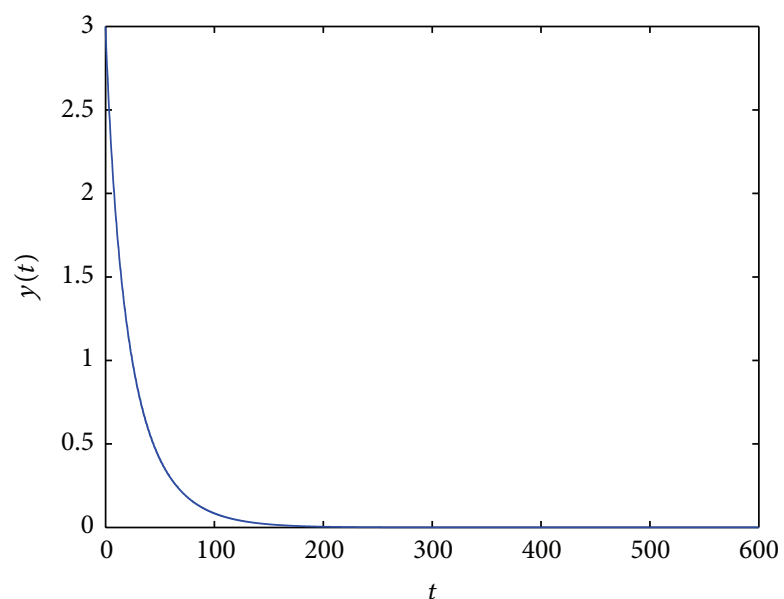

(b)

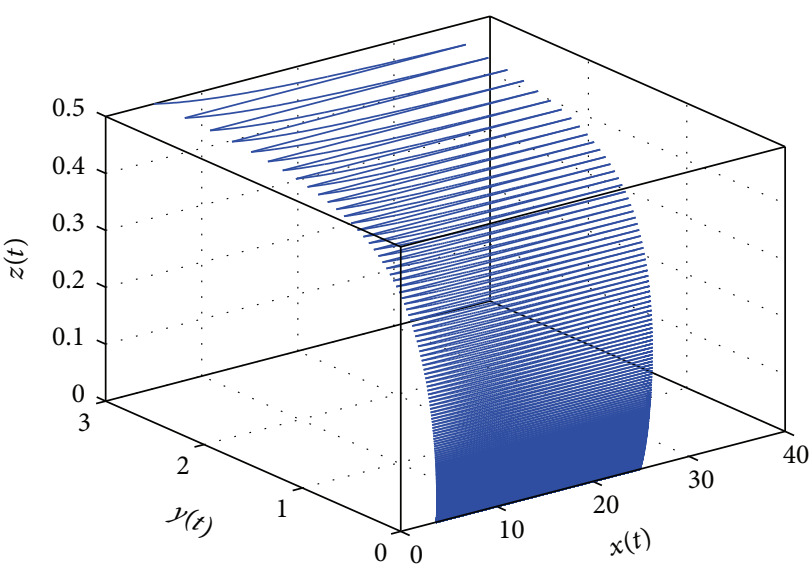

(d)

FIGURE 1: The dynamics of system (2) with the impulsive control strategy with $x(0)=5, y(0)=3$, and $z(0)=0.5$ and $r_{1}=0.98, r_{2}=0.05$, $d_{1}=0.001, d_{2}=0.01, a_{1}=0.02, a_{2}=0.03, a_{3}=0.01, e_{1}=4, e_{2}=0.8, e_{3}=2.131, b_{1}=30, b_{2}=50, b_{3}=1.5, m=0.02, \tau=0.1, \delta=0.856$, and $T=2$.

Case 2. If we choose $r_{1}=1.96, r_{2}=0.05, d_{1}=0.3, d_{2}=0.01$, $a_{1}=2, a_{2}=0.05, a_{3}=0.01, e_{1}=4, e_{2}=2, e_{3}=2.660201$, $b_{1}=20, b_{2}=16, b_{3}=10, m=0.02, \tau=0.5, \delta=0.8$, and $T=20$ with initial conditions $x(0)=5, y(0)=3$, and $z(0)=0.5$, which satisfies the condition of Theorem 10 , then from the time-series diagram of $x(t), y(t)$, and $z(t)$ we can see that the system is permanent, and all of the population can coexist in this case. From the phase portrait (Figure 2(d)) we can see there is a periodic solution. Moreover, if we set the economic threshold level ETL $=1.2$ (which is much less than the initial value $x(0)=5$ ), and from Figure 2(a), we can see that the pests population is less than ETL ultimately. That is to say, our control strategy is effective.

On the other hand, the selection of the economic threshold level (ETL) is closely related to the dynamical behavior of the pest population, especially to the maximum population after a period. Therefore, when the conditions of the permanent theorem (Theorem 10) hold, from the first equation of system (2), we can change some of the parameters of system (2) to decrease the economic threshold level, such as decreasing the value of parameter $r_{1}$ or increasing the value of parameter $d_{1}$. To verify this point, we consider the following Case 3.

Case 3. If we choose $r_{1}=1.86<1.96, d_{1}=0.36>0.3$ while the other values keep the same as Case 2, and plot the time-series of the pest population (see Figure 3(a)), it is obvious to see that the pest population can be controlled under a new ETL $=1.05$, which is lower than the previous ETL $=1.2$. Furthermore, multiple periodic solutions or periodic oscillations appear from the phase portrait at the moment (see Figure 3(b)).

\section{Conclusions and Discussions}

In this paper, we investigate the dynamics of a threedimensional food-chain system incorporating digest delay and periodic harvesting for the prey. The value of our study 


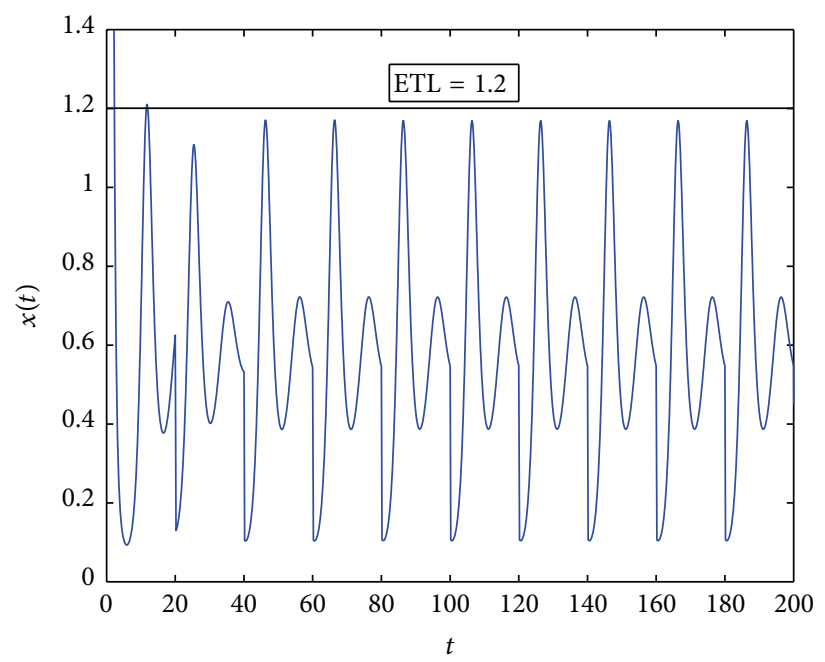

(a)

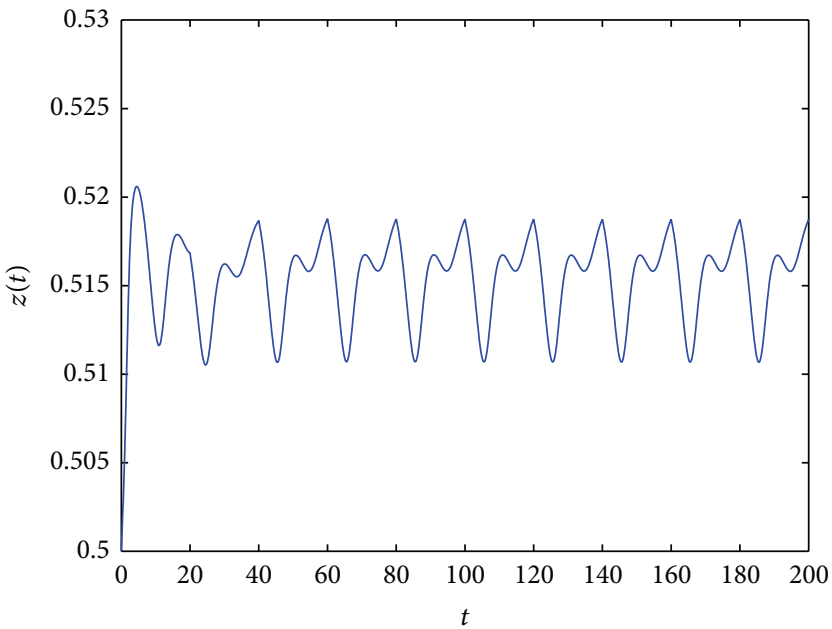

(c)

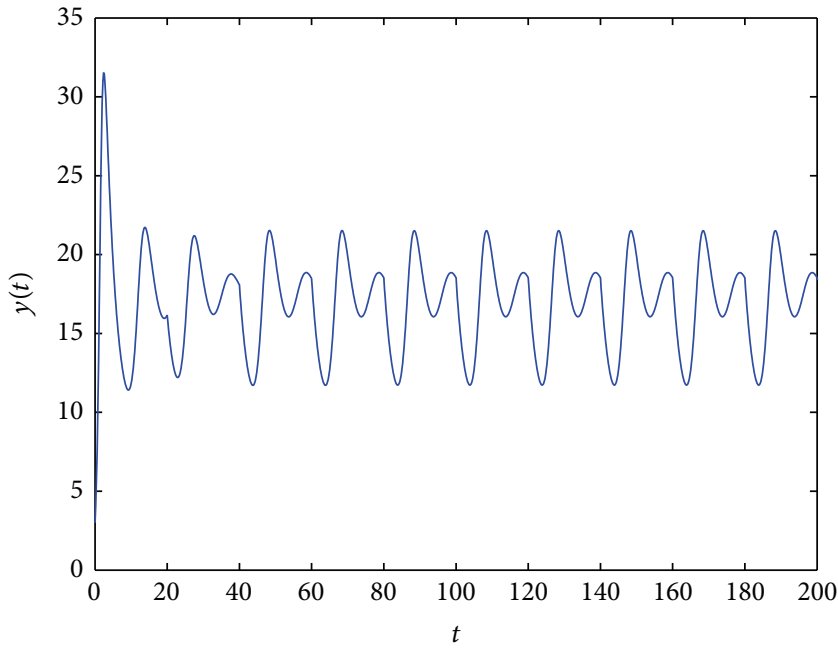

(b)

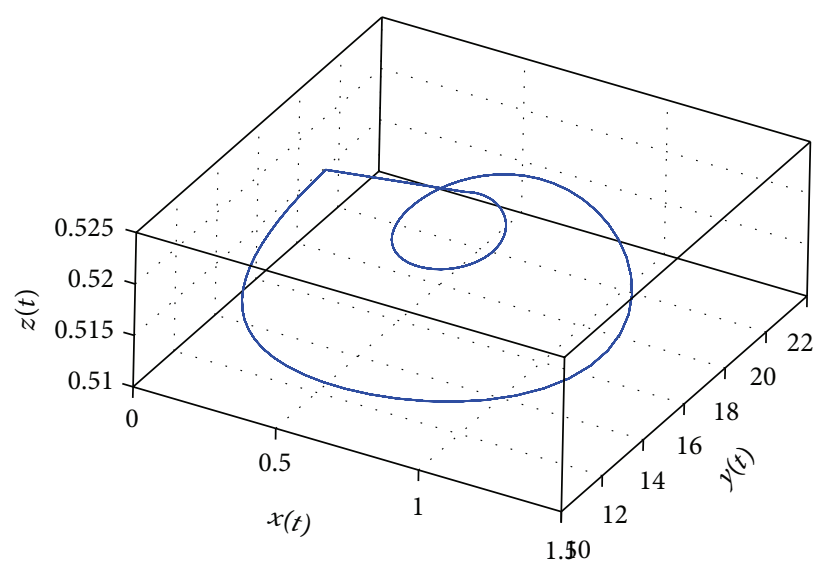

(d)

FIGURE 2: The dynamics of system (2) with the impulsive control strategy with $x(0)=5, y(0)=3$, and $z(0)=0.5$ and $r_{1}=1.96, r_{2}=0.05$, $d_{1}=0.3, d_{2}=0.01, a_{1}=2, a_{2}=0.05, a_{3}=0.01, e_{1}=4, e_{2}=2, e_{3}=2.660201, b_{1}=20, b_{2}=16, b_{3}=10, m=0.02, \tau=0.5, \delta=0.8$, and $T=20$.

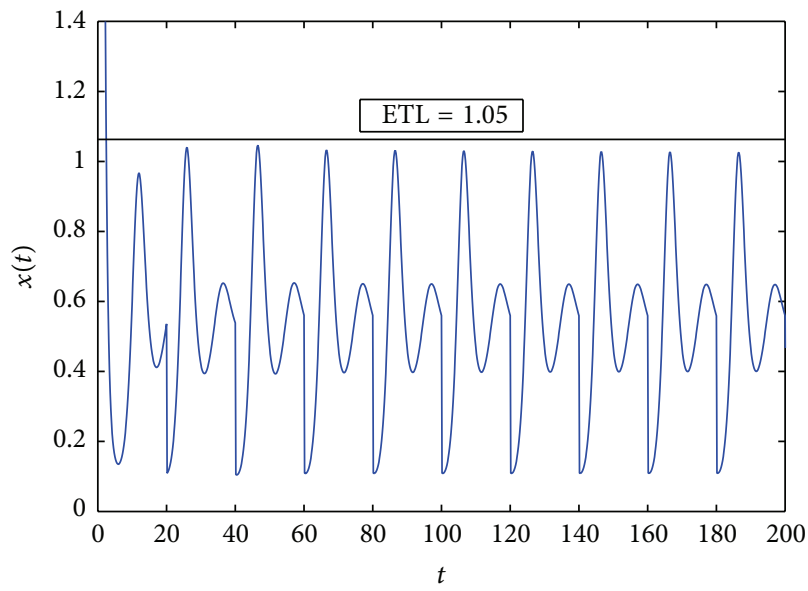

(a)

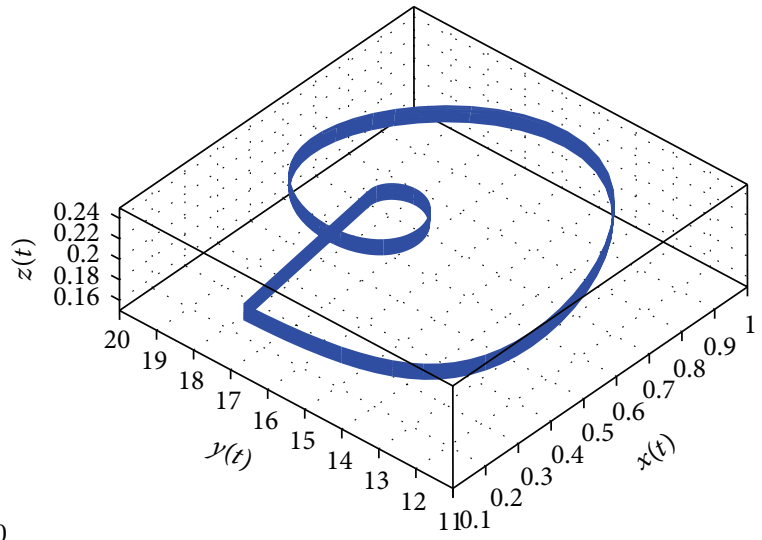

(b)

FIGURE 3: The dynamics of system (2) with the impulsive control strategy with $x(0)=5, y(0)=3$, and $z(0)=0.5$ and $r_{1}=1.86, r_{2}=0.05$, $d_{1}=0.35, d_{2}=0.01, a_{1}=2, a_{2}=0.05, a_{3}=0.01, e_{1}=4, e_{2}=2, e_{3}=2.660201, b_{1}=20, b_{2}=16, b_{3}=10, m=0.02, \tau=0.5, \delta=0.8$, and $T=20$. 
lies in two aspects: mathematically, we prove the existence of a predators-eradication periodic solution which is globally attractive and show that the pest population can be controlled under the economic threshold level (ETL) and the system can be uniformly permanent when the harvest period $T$ is long enough or the harvesting rate $\delta$ is not too large. Biologically, we succeed to find some strategies to control the population of the pests under a certain economic threshold level (ETL) and provide some reasonable suggestions for relevant ecological departments by these conclusions.

However, these control conditions are sufficient and tedious; then how to obtain some simpler and more extensive control conditions is desirable in future studies.

\section{Conflict of Interests}

The authors declare that there is no conflict of interests regarding the publication of this paper.

\section{Acknowledgments}

The authors would like to thank the anonymous referees for very helpful suggestions and comments which led to improvement of their original paper. This work is supported by the National Natural Science Foundation of China $(41072235,51349011)$ and Scientific Research Fund of Sichuan Provincial Education Department (11ZB192, 14ZB0115).

\section{References}

[1] W. Qin, G. Tang, and S. Tang, "Generalized predator-prey model with nonlinear impulsive control strategy," Journal of Applied Mathematics, vol. 2014, Article ID 919242, 11 pages, 2014.

[2] J. C. Van Lenteren and J. Woets, "Biological and integrated pest control in greenhouses," Annual Review of Entomology, vol. 33, no. 4, pp. 239-250, 1988.

[3] S. Tang and L. Chen, "Modelling and analysis of integrated pest management strategy," Discrete and Continuous Dynamical Systems B, vol. 4, no. 3, pp. 759-768, 2004.

[4] S. Tang, Y. Xiao, L. Chen, and R. A. Cheke, "Integrated pest management models and their dynamical behaviour," Bulletin of Mathematical Biology, vol. 67, no. 1, pp. 115-135, 2005.

[5] H. Guo and L. Chen, "The effects of impulsive harvest on a predator-prey system with distributed time delay," Communications in Nonlinear Science and Numerical Simulation, vol. 14, no. 5, pp. 2301-2309, 2009.

[6] H. Yu, S. Zhong, M. Ye, and W. Chen, "Mathematical and dynamic analysis of an ecological model with an impulsive control strategy and distributed time delay," Mathematical and Computer Modelling, vol. 50, no. 11-12, pp. 1622-1635, 2009.

[7] H. Yu, S. Zhong, R. P. Agarwal, and L. Xiong, "Species permanence and dynamical behavior analysis of an impulsively controlled ecological system with distributed time delay," Computers \& Mathematics with Applications, vol. 59, no. 12, pp. 3824-3835, 2010.

[8] L. Chen and F. Chen, "Dynamic behaviors of the periodic predator-prey system with distributed time delays and impulsive effect," Nonlinear Analysis: Real World Applications, vol. 12, no. 4, pp. 2467-2473, 2011.
[9] X. Meng, J. Jiao, and L. Chen, "The dynamics of an age structured predator-prey model with disturbing pulse and time delays," Nonlinear Analysis: Real World Applications, vol. 9, no. 2, pp. 547-561, 2008.

[10] H. Zhang, P. Georgescu, and L. Chen, "An impulsive predatorprey system with Beddington-DeAngelis functional response and time delay," International Journal of Biomathematics, vol. 1, no. 1, pp. 1-17, 2008.

[11] X. Meng, Z. Li, and J. J. Nieto, "Dynamic analysis of MichaelisMENten chemostat-type competition models with time delay and pulse in a polluted environment," Journal of Mathematical Chemistry, vol. 47, no. 1, pp. 123-144, 2010.

[12] M. Sekiguchi and E. Ishiwata, "Dynamics of a discretized SIR epidemic model with pulse vaccination and time delay," Journal of Computational and Applied Mathematics, vol. 236, no. 6, pp. 997-1008, 2011.

[13] H. Cheng and T. Zhang, "A new predator-prey model with a profitless delay of digestion and impulsive perturbation on the prey," Applied Mathematics and Computation, vol. 217, no. 22, pp. 9198-9208, 2011.

[14] H. Yu, S. Zhong, and R. P. Agarwal, "Mathematics analysis and chaos in an ecological model with an impulsive control strategy," Communications in Nonlinear Science and Numerical Simulation, vol. 16, no. 2, pp. 776-786, 2011.

[15] V. Lakshmikantham, D. D. Bainov, and P. S. Simeonov, Theory of Impulsive Differential Eqations, World Scientific, Singapore, 1989.

[16] D. D. Bainov and P. S. Simeonov, Impulsive Differential Equations: Periodic Solutions and Applications, Longman Scientific and Technical, Harlow, UK, 1993.

[17] Y. Kuang, Delay Differential Equations with Applications in Population Dynamics, Academic Press, San Diego, Calif, USA, 1993. 


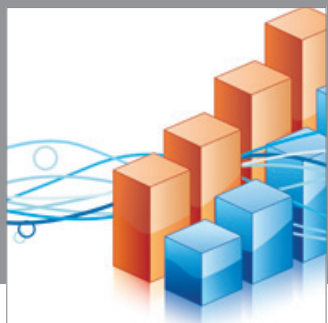

Advances in

Operations Research

mansans

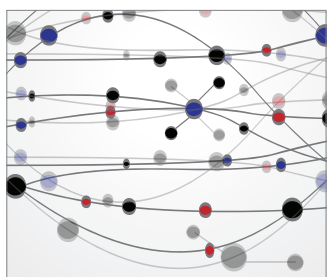

The Scientific World Journal
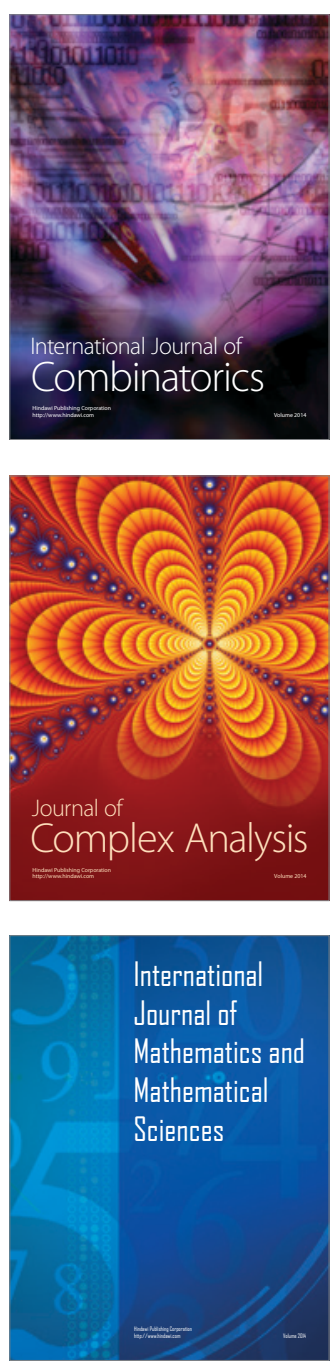
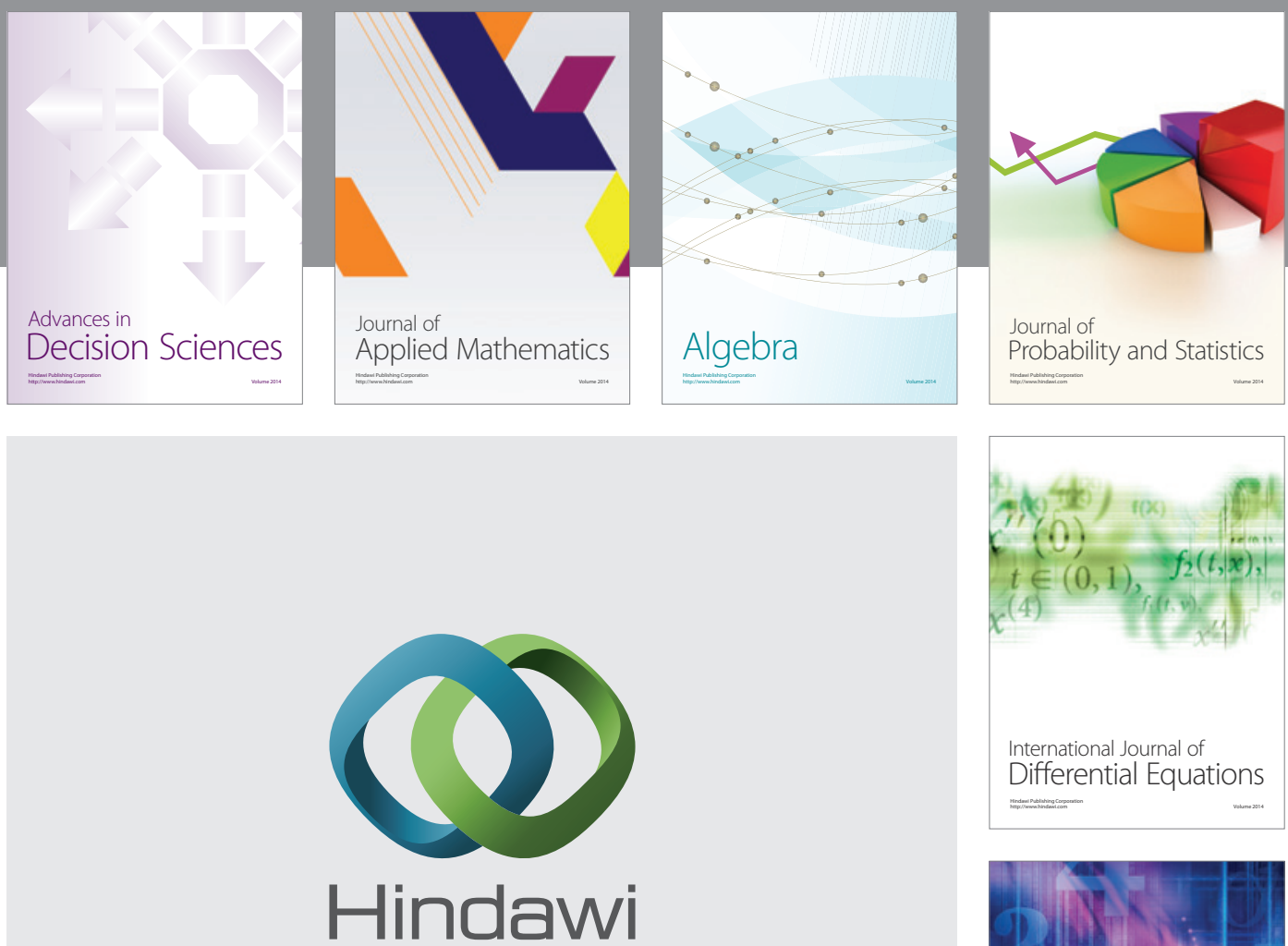

Submit your manuscripts at http://www.hindawi.com
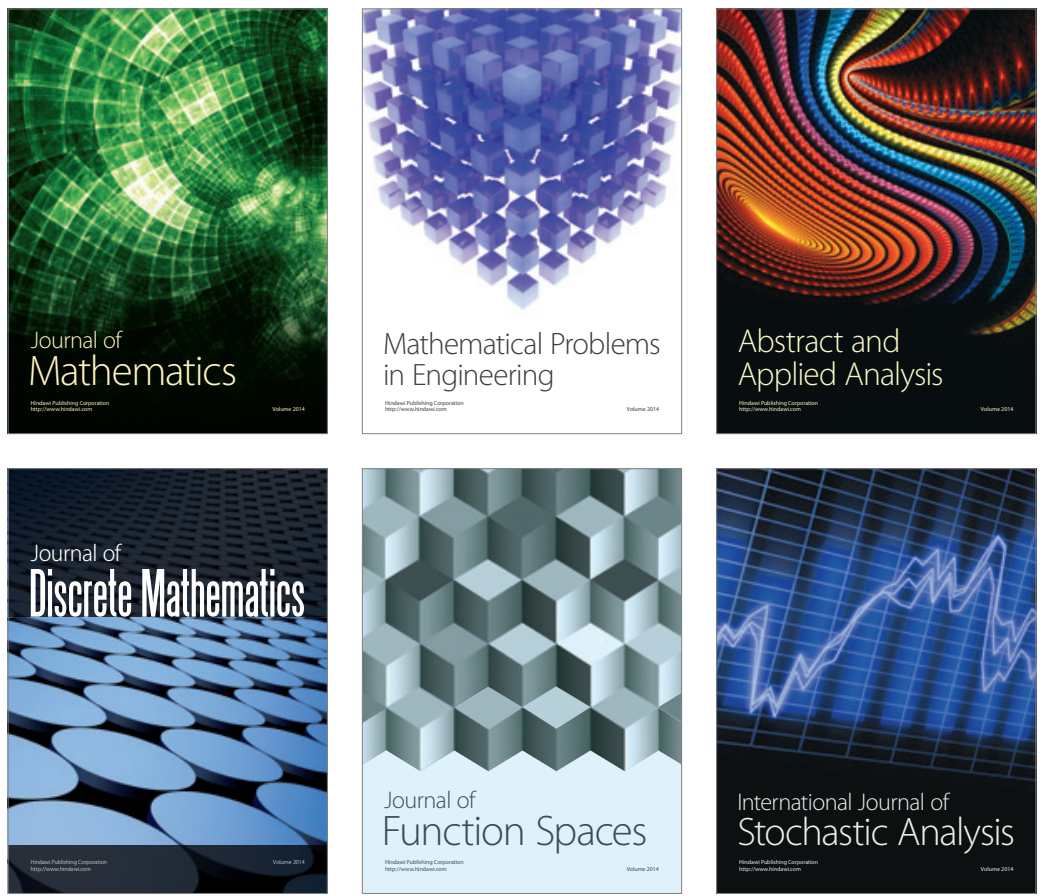

Journal of

Function Spaces

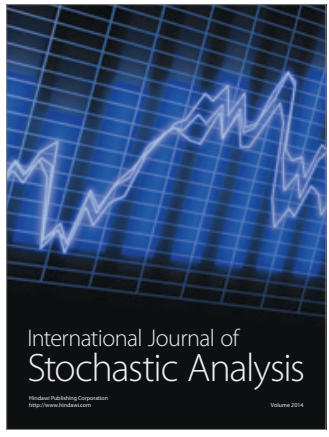

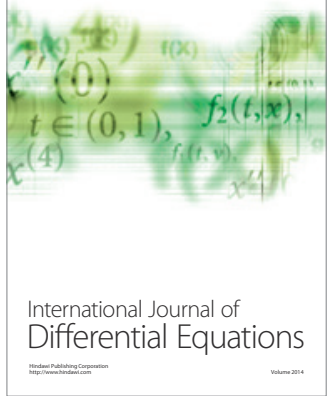
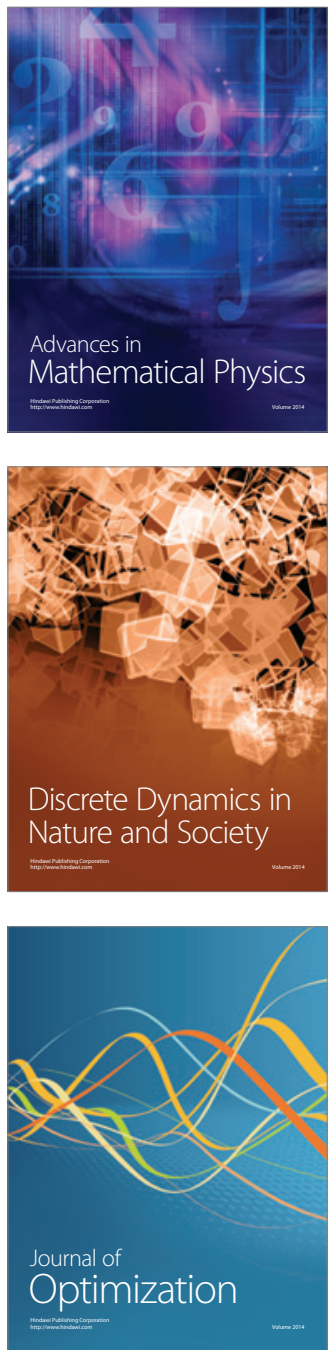